\title{
MANAGEMENT OF ORAL RECURRENT APHTHOUS STOMATITIS (ORAS)
}

Khoo S.P. Management of oral recurrent aphthous stomatitis (ORAS). Annal Dent Univ Malaya 1999; 6: 40 42

\section{ABSTRACT}

Oral recurrent aphthous stomatitis is the most common oral mucosal disease. Despite much clinical and research attention, the causes remain incompletely understood. Treatment options include no treatment, treatment of associated systemic diseases, topical medications, systemic treatment and palliative treatments. The most effective treatments involve agents that suppress or modulate the immune function. Topical agents are preferred due to its limited side-effects. Adjunct pain control and prevention of secondary infections is sometimes necessary.

Key words: - Oral - recurrent stomatitis - management.

\section{INTRODUCTION}

Oral recurrent aphthous stomatitis (ORAS) is a very common oral mucosal disorder of unknown aetiology. To date the causes remain poorly understood; ORAS is not entirely preventable and thus there is no specific management for it.

\section{GENERAL MANAGEMENT}

Management efforts are directed towards reducing the symptoms, diminishing the lesion size and duration and in particular prolonging the ulcer-free periods. Before definitive therapy is instituted it is important to manage any existing underlying disorder that might contribute to ORAS such as any underlying systemic disorders and the presence of any triggering factors of ORAS (1).

If stress is felt to be a strong cofactor, consultation with a behavioral therapist or a psychologist/psychiatrist or the use of relaxation techniques will be helpful. A gluten-free diet is advocated for patient's gluten-enteropathy. Replacement therapy with Iron, Folic acid, vitamins B6 and B12 are used for patients with underlying haematological deficiencies.

\section{PHARMACOLOGICAL THERAPY}

Pharmacological therapies that have been used have centered around topical drug and sometimes systemic therapy. Topical therapies include animicrobials, analgesic mouth rinses and gels, topical glucocorticosteroids, immunomodulators and hormones.

The most common topical therapy uses glucocorticoids such as hydrocortisone, triamcinolone, flucinonide, betamethasone and flumethasone (2). These medications reduce symptoms and will not cause hypothalamic-pituitary-adrenal axis suppression when used for less than 3 weeks. Administration of corticosteroids in single daily doses compared with multiple daily doses and on alternate-day regimens will help minimize the suppression (3). The corticosteroids can be applied in the form of solutions, creams, gels, aerosols, perilesional infiltrations or lozenges to be dissolved in the

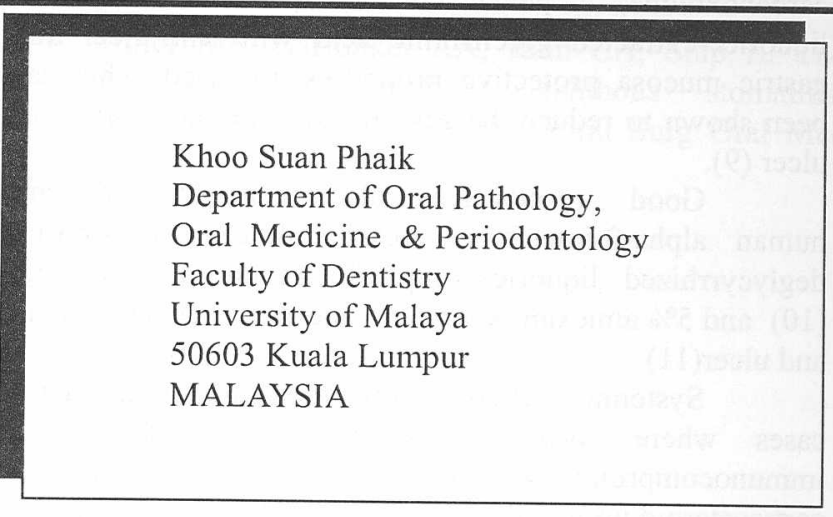

mouth. The results are particularly beneficial especially when application is started in the initial stages of the ulceration.

Triamcinolone $(0.05-0.1 \%)$ and fluocinolone (0.05-0.1\%) cream applied 3-5 times a day can reduce ulcer size and number and prolong the ulcer-free period. Vincent \& Lilly (4) recommended the use of topical triamcinolone acetonide $0.1-0.2 \%$ aqueous suspension, $5 \mathrm{ml}$ oral rinse and expectorate 4 times daily. Betamethasone 17-benzoate gel improves the symptoms and duration of lesion but for less accessible areas, beclomethasone spray may be useful.

Greater efficacy can be achieved with stronger corticosteroids that are administered for long-lasting, nonhealing or major ORAS. For example, clobetasol propionate ointment $0.05 \%$ in adhesive paste used $2-3$ times daily in patients with persistent major ORAS showed complete remission in 5 of 7 patients; no major side-effects was detected (5).

In more recalcitrant cases systemic prednisolone are used, where a 2-week tapering dose is employed, starting with $60 \mathrm{mg}(6)$. The addition of a steroid - sparing drug such as Azathioprine have benefited patients.

Perilesional infiltrations using triamcinolone $\left(0.5 \mathrm{mg}\right.$ per $2.5 \mathrm{~cm}^{2}$ of lesion or $\left.5 \mathrm{mg} / \mathrm{ml}\right)$ or hydrocortisone $(25 \mathrm{mg} / \mathrm{ml})$ may be indicated for large, painful localised lesions which are slow to heal. Dosing may be repeated every 10-15 days for as long as the lesions persist.

Studies using antimicrobial rinses such as tetracycline and chlorhexidine gluconate have reported a reduction in the number of ulcer days and severity of the ulcers, increase in ulcer-free days but have not been able to prevent recurrences(2). In a more recent study(7) Triclosan, an antibacterial agent introduced in toothpastes and mouthrinses, have been shown to reduce significantly the number of ulcers. The severity and frequency of ulcers were also reduced. This has been attributed to the antiinflammatory effect of the agent.

Analgesic mouthrinses containing benzydamine hydrochloride, an indole-group non-salicylic antiinflammatory analgesic are also used as a $0.15 \%$ rinse 3 times a day although a study has found little value of chlorhexidine mouthwash over placebo in the management of ORAS (8). Also beneficial is dexamethasone elixir $(0.5 \mathrm{mg} / 5 \mathrm{ml})$, diphenydramine elixir $(12.5 \mathrm{mg} / 5 \mathrm{ml})$ and 
dyclonine hydrochloride $0.5 \%$ or $1.0 \%$ can be used $3-4$ times daily.

In some centres around the world, disodium carbenoxolone, a pentacyclic triterpene derived from liquorice-extracted glycirrhinic acid with anti-ulcer and gastric mucosa protective properties are used. This has been shown to reduce the severity of pain and number of ulcer (9).

Good results have also been achieved with human alpha-2-interferon cream, topical cyclosporin, deglycyrrhized liquorice, topical 5-aminosalicylic acid (10) and 5\% amlexanox which reduces the erythema, pain and ulcer(11).

Systemic corticosteroids are mostly reserved for cases where local measures have failed or in immunocompromised patients(12). The commonest corticosteroid used is Prednisolone eg. $20 \mathrm{mg}$ may be given 2 times daily for 5 days and then once daily for a further week (4) or a starting dose of $60 \mathrm{mg}$ and tapering down in 2 weeks.

Other immunosuppressive drugs such as colchicine, cyclosporin and thalidomide have been used to treat ORAS. Cyclosporin suppresses lymphocytedependent-antibody response. Colchicine reduces neutrophil chemotaxis and has been employed in the management of major ORAS and at a dose of $0.6 \mathrm{mg} 3$ times daily where remission was maintained so long as the drug is maintained (13). A dose of $1.5 \mathrm{mg} /$ day of colchicine for 2 months has been shown to reduce the frequency and pain of ORAS (14).

The use of thalidomide for ORAS has been reported since the late 70 's. Thalidomide with recommended dose of up to $100 \mathrm{mg}$ /day for $2-3$ months, has been shown to inhibit histamine-induced circulating immune-complex mediated damage (15). Thalidomide has found its use particularly in recalcitrant lesions and in HIV-infected patients. The side effects, especially its teratogenic potential, should be taken into account.

Combination drugs using colchicine and thalidomide have been employed with initial doses of 100-300mg thalidomide combined with 1-3mg colchicine. The development of new lesions was controlled and recurrences was prevented (10)). A maintenance dose of $50-100 \mathrm{mg}$ thalidomide with $1 \mathrm{mg}$ colchicine have been recommended (14).

Immunopotentiating agents such as levamisole, which enhances cell and humoral-mediated immunity have been found to be beneficial in ORAS patients. However, due to its controversial results and the side effects of levamisole such as hyperosmia, dysgeusia and agranulocytosis, its use is not indicated (16). Beneficial effects have been reported with a number of other drugs $(6,10,12)$ such as AM3, a biological response modifier which markedly reduces the ulcer number and duration after 6 months of therapy. Pentoxyphilline (400mg daily 8 hourly for 6 months) has been reported to eliminate outbreaks of ORAS in $80 \%$ of affected patients but lesions have been shown to recur after treatment is stopped. Azelastine hydrochloride helps stabilises cell membranes and suppresses reactive oxygen generation; oral administration of $1 \mathrm{mg} 2$ times daily for 3 weeks has been shown to reduce significantly the frequency of occurrence, the ulcer duration and oral irritation in 43 patients.

Other drugs such as transfer factor, gammaglobulin, recombinant human interferon alpha-2a, monoamine oxidase inhibitors, cromoglycates, isoprinosine, dapsone, zinc sulphate, prostaglandin E-2, acyclovir, lasers (CO2 and Helium-Neon) and LongoVital, a food supplement are amongst the many therapeutic modalities reported to have beneficial effects in the treatment of ORAS (10). However, besides the high costs in some cases and its side effects, more importantly, the lack of comprehensive clinical testing and standardisation poses a problem.

In summary, the management of ORAS remains non-specific until a principal causal factor can be isolated Research has been, and will continue to be geared towards this end.

\section{REFERENCES}

1. Rogers RS. Recurrent aphthous stomatitis : clinical characteristics and associated systemic disorders. Semin Cutan Med Surg 1997; 16(4) : 278-83.

2. Scully C, Porter SR. Recurrent aphthous stomatitis current concepts of aetiology, pathogenesis and management. J Oral Pathol Med 1989; $18: 21-7$.

3. Helfer EL, Rose LI. Corticosteroids and adrenal suppression : characterizing and avoiding the problem. Drugs $1989 ; 38: 838-45$.

4. Vincent SD, Lilly GE. Clinical, historic and therapeutic features of aphthous stomatitis. Literature review and open clinical trial employing steroids. Oral Surg Oral Med Oral Path 1992; $74: 79-86$

5. Lozada-Nur $\mathbb{F}$, Huang $\mathrm{MZ}$, Zhou GA. Open preliminary clinical trial of clobetasol propionate ointment in adhesive paste for treatment of chronic oral vesiculoerosive diseases. Oral Surg Oral Med Oral Path 1991; $71: 678-683$

6. Ship JA. Recurrent aphthous stomatitis : an update Oral Surg Oral Med Oral Path Oral Radiol Endod $1996 ; 81: 141-7$.

7. Skaare AB, Herlofson BB, Barkvoll P. Mouthrinses containing triclosan reduce the incidence of recurrent aphthous ulcers. J Clin Periodontol 1996; $23: 778$

8. Matthews RW, Scully CM, Levers BG, Hislop WS Clinical evaluation of benzydamine, chlorhexidine and placebo mouthwashes in the management of recurrent aphthous stomatitis. Oral Surg Oral Med Oral Path 1987; $63: 189-91$.

9. Poswillo D, Partridge M. Management of recurrent aphthous ulcers. A trial of carbenoxolone sodium mouthwash. Br Dent J 1984; 157 : 55-7.

10. Bagan J. Recurrent aphthous stomatitis. In Innovations and developments in non-invasive orofacial health care. Eds. Porter S, Scully C. Science Reviews, 1996; 75-95.

11. Khandwala A, Van Inwegen RG, Charney MR, Alfano MC. 5\% Amlexanox oral paste, a new treatment for recurrent minor aphthous ulcers. I. Clinical demonstration of acceleration of healing and resolution of pain. Oral Surg Oral Med Oral Pathol Oral Radiol Endod 1997; 83 : 222-30. 
12. MacPhail L. Topical and systemic therapy for recurrent aphthous stomatitis. Semin Cutan Med Surg 1997; 16 : 301-7.

13. Ruah CB, Stram JR, Chasin WD. Treatment of severe recurrent aphthous stomatitis with colchicine. Arch Otolaryngol Head Neck Surg 1988; 114: 671-675.

14. Katz J, Langevitz P, Shemer J, Barak S, Livneh A. Prevention of recurrent aphthous stomatitis with colchicine : an open trial. I Am Acad Dermatol 1994; 31: 459-61.
15. Revuz J, Guillaume JC, Janier M, et al. Crossover study of thalidomide vs placebo in severe recurrent aphthous stomatitis. Arch Dermatol 1990; 126 : 923 27.

16. Miller MF, Garfunkel AA, Ram CA, Ship II. The inheritance of recurrent aphthous stomatitis. Observations on susceptibility. Oral Surg Oral Med Oral Path $1980 ; 49: 409-412$. 